\title{
DIAGNÓSTICO DO PDP DE UM PROJETO DESENVOLVIDO EM EQUIPES VIRTUAIS PARA A CONSTRUÇÃO DE UM VENTILADOR PULMONAR DE BAIXO CUSTO
}

Roberto Canedo Rosa (engcanedorosa@gmail.com) - Universidade de Brasília, UNB.

Sanderson Cesar Macedo Barbalho ( $\underline{\text { sandersoncesar@unb.br) }}$ - Universidade de Brasília, UNB.

Guillermo Alvarez Bestard (guillermo@unb.br) - Universidade de Brasília, UNB.

Carlos Humberto Llanos Quintero (llanos@ unb.br) - Universidade de Brasília, UNB.

Fábio Ferreira Amorim (ffamorim@gmail.com) - Escola Superior de Ciências da Saúde, ESCS.

Fábio Henrique Monteiro Oliveira (1017591@etfbsb.edu.br) - Instituto Federal de Brasília, IFB.

Sérgio Henrique Evangelista (toddyprof @ufscar.br) - Universidade Federal de São Carlos, UFSCar.

\section{RESUMO}

Os projetos colaborativos entre governo, universidade e empresa privada desempenham um papel essencial no gerenciamento de crises, além de serem fundamentais para a implementação de estratégias de inovação. A partir das necessidades geradas pela crise de saúde pública advindas com a Covid-19, este trabalho apresenta uma análise do diagnóstico do processo de desenvolvimento de um ventilador pulmonar de baixo custo, desenvolvido em parceria entre pesquisadores da Universidade de Brasília (UNB), da Universidade Federal de São Carlos (UFSCAR), do Instituto Federal de Brasília (IFB), e da Escola Superior de Ciências da Saúde (ESCS). O projeto foi financiado pela Fundação de Apoio à Pesquisa do Distrito Federal, é gerenciado financeiramente pela Fundação de apoio da UNB, e tem parceria com uma empresa privada, com o foco em transferir tecnologia e na produção em escala do ventilador. O diagnóstico do PDP foi realizado a partir da associação de dois modelos amplamente difundidos na literatura, apresentando características peculiares de um projeto materializado em ambiente universitário, com prazos curtos, e atividades desenvolvidas, em grande parte, remotamente. Os resultados apresentados comprovaram uma estrutura similar a projetos do tipo mecatrônico, e com uma alta interação entre os atores do projeto na realização das atividades.

Palavras chave: Processo de desenvolvimento de produto; projeto colaborativo; ventilador pulmonar; Covid-19 


\section{INTRODUÇÃO}

As abordagens que modelam o Processo de Desenvolvimento de Produtos (PDP) oferecem uma sequência de atividades, organizadas em fases, que buscam definir as especificações do produto e do processo de produção, a partir de transformações de informações de mercado e tecnologia, e com o foco em atender às necessidades do consumidor (Pugh, 1991; Whellwright \& Clark, 1992; Rozenfeld et al., 2006).

Os modelos de referência para o PDP são considerados um conjunto de melhores práticas, sendo uma modelagem explícita e sistemática para apoiar a execução de tarefas aplicáveis ao mundo real. O PDP engloba todos os atores organizacionais afetados pelo desenvolvimento do produto, que envolve as atividades iniciais do planeamento estratégico até a retirada do produto do mercado, e seu respectivo descarte. Verifica-se, assim, uma integração essencial entre as partes envolvidas em diferentes áreas que estabelece, por sua vez, uma conexão distinta com o produto, a partir das diversas perspectivas de cada ator (Barbalho, 2006).

As colaborações em projetos de desenvolvimento de produtos entre instituições e atores públicos e privados, apresentam grande potencial de responder com eficácia a eventos extremos como a pandemia global do COVID-19 que se espalhou implacavelmente. As colaborações entre o governo, a universidade, e a empresa privada desempenham um relevante papel para o gerenciamento de crises buscando capturar experiências, capacidades, recursos e conhecimentos, compartilhar informações, reunir recursos e coordenar esforços com o foco de recuperação em uma emergência (Huanming Wang et al., 2021; Quayle et al., 2019).

Os projetos realizados a partir da interação entre instituições públicas e privadas apresentam complexidades singulares, sendo cientificamente amparado pelo modelo de hélice tríplice, que por sua vez sustentam os sistemas de inovação. Cada ator da hélice tríplice desempenha um papel fundamental no processo colaborativo, e a partir dessa interação é possível estimular o aprendizado e direcionar avanços em tecnologias, contribuindo para a implementação de estratégias de inovação, e oferecendo novas oportunidades de investigação científica (Lemos, 2013; Valente, 2010).

O presente trabalho tem como principal enfoque o diagnóstico do processo de desenvolvimento de um ventilador pulmonar mecânico de baixo custo para suporte à vida de pacientes acometidos pela COVID-19. O foco é entender o andamento de um projeto de parceria entre universidade, estado e o setor privado, realizado de maneira virtual com 
parceiros dispersos geograficamente, auxiliando, assim, na sistematização do PDP e documentando as características, estruturas, e atividades dos diversos atores envolvidos.

\section{PROJETO COLABORATIVO GOVERNO-UNIVERSIDADE-INDÚSTRIA}

O apoio a pesquisas, os projetos colaborativos, a transferência de conhecimento, e a transferência de tecnologia, são alternativas de cooperação entre a universidade, o governo e a empresa privada (Santoro \& Chakrabarti, 2002). Os sistemas de ciência e tecnologia, exercem um papel fundamental nas economias baseadas em conhecimento, e são fortalecidos pelos projetos de cooperação entre empresas, governo e universidades, adquirindo, assim, alto impacto para as demandas sociais em diversas áreas como a educação, meio ambiente, e a saúde (Lastres \& Cassiolato, 2000).

Todos os agentes do processo colaborativo desempenham papéis fundamentais para o êxito da interação. Para tanto, é necessário definir, de maneira objetiva, os papeis de cada parceiro e suas respectivas responsabilidades. Para a criação de redes de colaboração estáveis são necessários apontamentos direcionadores: a política de interação do organismo de pesquisa e o ambiente industrial; a conduta da interação durante toda a vida do projeto; a preparação da transferência dos resultados; a negociação e as condições burocráticas dos contratos; a conduta da transferência; a harmonização das representações dos parceiros; e a interação durante o processo de industrialização dos resultados (De Vasconcelos \& Ferreira, 2000).

A interação entre a universidade e a empresa privada tornou-se um pré-requisito para a consolidação de avanços tecnológicos, sendo um motor propulsor em países desenvolvidos para alavancar inovações, indispensáveis no aumento vertiginoso da competitividade no mercado global (Noveli \& Segatto, 2012). As colaborações público-privadas, além disso, tem um potencial de responder com eficácia a crises sanitárias e de saúde pública, como a COVID-19, desenvolvendo diversos arranjos nos quais produtos e serviços podem ser fornecidos com base no compartilhamento de riscos, recursos, custos e benefícios (Huanming Wang et al., 2021; Bryson et al., 2006).

Geralmente, o produto desenvolvido pelo processo colaborativo, entre os agentes públicos e privados, tem relações contratuais detalhadas com liderança de um ator governamental com vontade política para estímulo à criação (Kapucu, 2012). 


\section{METODOLOGIA}

O presente trabalho trata de um estudo de caso (Yin, 2001), descritivo, qualitativo, com aplicação do Framework proposto por Barbalho e Rozenfeld (2004) em um projeto de desenvolvimento de produtos para o contexto da COVID-19. Os dados foram obtidos através de observação participante no projeto e análise documental dos relatórios de progresso.

A escolha do projeto se deu pela facilidade de acesso aos dados e pelo interesse da equipe em melhorar o seu PDP. Assim, foi possível acessar informações relativas à hierarquia do projeto, identificação dos responsáveis pelas tomadas de decisões, e a execução das atividades ao longo do desenvolvimento. Foi ainda possível acessar os documentos gerados em cada fase do projeto, identificando sub-etapas e movimentações relacionadas ao desenvolvimento do produto.

O framework de pesquisa utilizado foi escolhido em função de haver literatura anterior utilizando-o, o que permitiria um comparativo dos resultados desse estudo com as aplicações anteriores desse framework.

\section{FRAMEWORK DE DIAGNÓSTICO DO PDP}

O trabalho apresentado baseou-se no referencial de diagnóstico do Processo de Desenvolvimento de Produtos (PDP) proposto por Whellwright e Clark (1992), associado ao padrão de descrição do PDP com o foco na modelagem dos processos de negócios transcritos por Rozenfeld et al. (2006). O framework de avaliação para o diagnóstico do PDP é apresentado na Figura 1. 


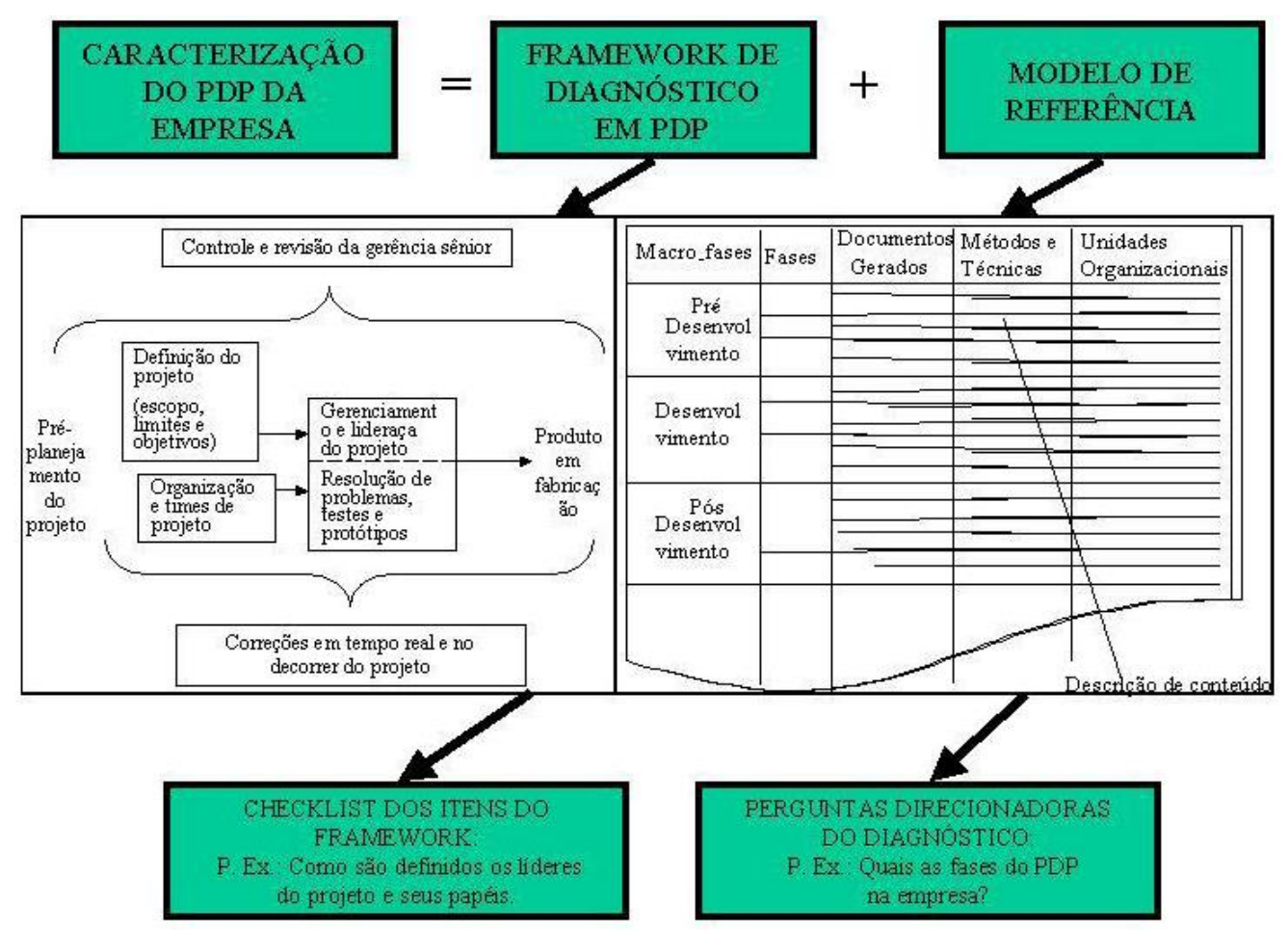

Figura 1 - Framework de diagnóstico do PDP. Fonte: Barbalho e Rozenfeld (2004).

Para Whellwright e Clark (1992) o processo de desenvolvimento é um conjunto complexo de atividades que se estende por um período de tempo considerável, e como ponto de partida para compreender essa complexidade e identificar as escolhas críticas da alta administração, integram-se seis elementos, ou dimensões, para criar um padrão de detalhamento de desenvolvimento, incluindo a definição do projeto, a organização e os times, o gerenciamento e liderança do projeto, a resolução de problemas, testes e protótipos, o controle e revisão da gerência sênior e as correções em tempo real e no decorrer do projeto.

No modelo de Rozenfeld et al. (2006) o PDP é dividido em macro-fases, que por sua vez se divide em fases, que ainda podem ser detalhados em atividades e tarefas. A cada fase são alocados novos elementos caracterizadores com o foco em apresentar indicativos para a gestão do PDP. Na macrofase de pré-desenvolvimento o objetivo é garantir que o portfólio de projeto esteja em consonância com as oportunidades do mercado, as restrições envolvidas e a estratégia da empresa. Na etapa de desenvolvimento, o destaque é o projeto do produto englobando as etapas de projeto informacional, conceitual, detalhado, a preparação da produção e seu respectivo lançamento. $\mathrm{Na}$ etapa de pós-desenvolvimento realiza-se o acompanhamento sistemático do produto no mercado, e a avaliação das melhorias necessárias no ciclo de vida do produto. 


\section{ESTUDO DE CASO}

\subsection{PROJETO VENTILADOR PULMONAR DE BAIXO CUSTO}

No projeto do ventilador pulmonar de baixo custo o agente governamental atua a partir de uma agência de financiamento, a universidade tem um papel fundamental sendo o agente principal do projeto, transformando os recursos em conhecimento científico e aplicando os modelos de referência para a produção de protótipos e do produto tecnológico, o ventilador. A empresa acompanha todo o projeto técnico compartilhando conhecimentos e amparando as soluções de engenharia com fins de sustentar a transferência tecnológica e a produção em escala do produto.

A ventilação mecânica invasiva, definida como a liberação de pressão positiva nos pulmões por meio de um tubo endotraqueal ou de traqueostomia (Carvalho; Toufen junior; Franca, 2007) tornou-se um procedimento essencial para os cuidados de suporte à vida em pacientes acometidos pela COVID-19. Um desafio decorrente da crise de saúde pública mundial foi a escassez dos ventiladores mecânicos pulmonares nos serviços de saúde. Portanto, viabilizar a produção e o projeto de ventiladores pulmonares, tornou-se um direcionamento de pesquisas e de parcerias entre instituições em diversos países.

O projeto do ventilador pulmonar foi iniciado logo no início da pandemia no Brasil, a partir da necessidade dos leitos de UTI. O projeto foi viabilizado em meados de junho de 2020, por meio de um edital financiado pelo FAP-DF e como órgão gestor financeiro a FINATEC, que dá o suporte na compra dos equipamentos. O projeto em execução foi impulsionado pela parceria entre professores-pesquisadores-gestores de universidades brasileiras. Uma parceria com uma empresa privada foi realizada com o objetivo da transferência tecnológica e para a continuidade de produção do equipamento desenvolvido.

A estrutura organizacional do projeto é composta por professores, alunos de graduação, mestrandos e doutorandos de diversas áreas, incluindo engenharias e saúde. Por se tratar de uma equipe pequena, de alta capacitação acadêmica e profissional e de grande flexibilidade, sua estrutura é moldada por áreas específicas de atuação. Na Figura 2, visualizase a estrutura simplificada de pesquisa e desenvolvimento do projeto. 


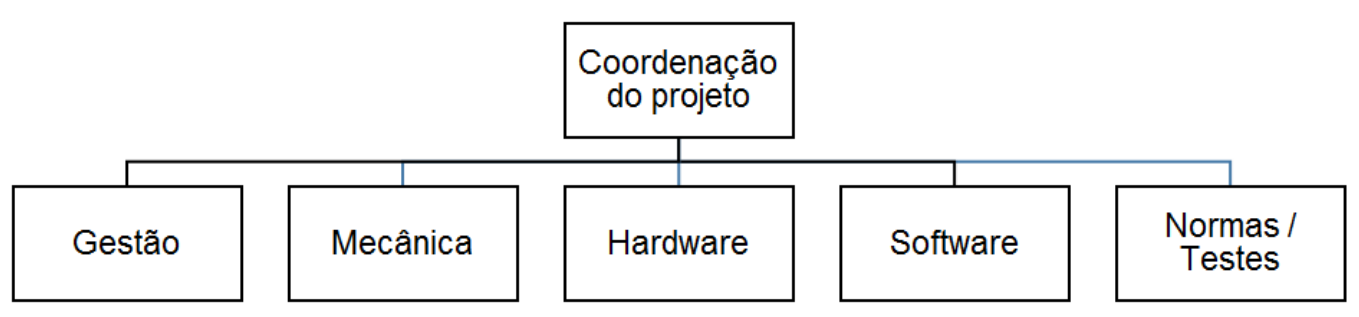

Figura 2- Estrutura de Pesquisa e Desenvolvimento do projeto.

O time de gestão é responsável pela entrega de cronogramas, da matriz de responsabilidade, dos registros em atas, do levantamento de requisitos funcionais, da rastreabilidade de itens, dos orçamentos, do controle de aquisição de itens de infra-estrutura, e da gestão da documentação de engenharia. A equipe de mecânica é responsável pelo projeto mecânico, desenho mecânico, montagem mecânica, aquisição e fabricação de peças, e montagem da bancada para testes. O time de hardware é responsável pelo projeto eletrônico com software embarcado e a interface entre as partes mecânicas e o software, além disso, incluem-se o projeto do sistema de controle, do acionamento, e do sistema de energia e medição. A equipe de software é responsável pelo desenvolvimento do software de operacionalização, pelo desenho do display, dos ciclos de alarmes e botões, e da avaliação de usabilidade do equipamento. O time de normas e testes é responsável por verificar se o produto atende as diretrizes da ANVISA.

\subsection{CARACTERIZAÇÃO DO PDP DO PROJETO}

O projeto do ventilador pulmonar de baixo custo ainda está em curso, e como não se trata de uma empresa já consolidada, e sim de um projeto de parceria entre instituições por meio de um processo colaborativo, e desenvolvido de maneira remota, as estruturas do PDP apresentam uma fluidez, que se ajustam no decorrer do projeto. O Quadro 1 apresenta os principais elementos identificados na análise do projeto mediante o framework desenvolvido por Whellwright e Clark (1992). 
Quadro 1- Síntese da análise do projeto baseado no modelo de Whellwright e Clark (1992).

\begin{tabular}{|c|c|}
\hline $\begin{array}{l}\text { ELEMENTO DO } \\
\text { FRAMEWORK }\end{array}$ & CARACTERÍSTICAS \\
\hline $\begin{array}{l}\text { Definição do } \\
\text { projeto }\end{array}$ & $\begin{array}{l}\text { O desenvolvimento do produto ventilador pulmonar de baixo custo foi } \\
\text { formatado a partir de um planejamento estratégico de curto prazo, onde há uma } \\
\text { definição de suas premissas atendidas por um edital de financiamento. A } \\
\text { necessidade do mercado foi avaliada, o que legitimou a importância do projeto } \\
\text { para garantir a segurança da disponibilidade do equipamento, que será fabricado } \\
\text { com o foco nos leitos de UTI no Brasil. Essas informações foram identificadas } \\
\text { por uma equipe de professores/cientistas, sendo sua proposta elaborada com } \\
\text { base em um projeto colaborativo e de parceria entre instituições pública e } \\
\text { privada. A equipe dos professores tem autonomia para ajustar o escopo do } \\
\text { projeto desde que não altere o objeto do contrato de financiamento que é o } \\
\text { ventilador de baixo custo para utilização em UTI. }\end{array}$ \\
\hline $\begin{array}{l}\text { Organização e } \\
\text { times de projeto }\end{array}$ & $\begin{array}{l}\text { A estrutura organizacional do projeto é composta por professores, especialistas, } \\
\text { alunos de graduação, mestrandos e doutorandos de diversas áreas, incluindo } \\
\text { engenharias e saúde. Cada área é vinculada a um professor de magistério } \\
\text { superior, que tem por responsabilidade garantir a fluidez e o cumprimento da } \\
\text { evolução do projeto. Os membros de cada equipe são alocados conforme as } \\
\text { necessidades e interesse de cada professor. A estrutura organizacional matricial } \\
\text { básica do projeto compreende a coordenação, o time de gestão, o time de } \\
\text { mecânica, o time de hardware, e o time de software. Estrutura essa similar a } \\
\text { projetos do tipo mecatrônico. }\end{array}$ \\
\hline $\begin{array}{l}\text { Gerenciamento e } \\
\text { liderança do } \\
\text { projeto }\end{array}$ & $\begin{array}{l}\text { O gerenciamento das etapas de desenvolvimento do produto é realizado pelo } \\
\text { gestor do projeto juntamente com os professores responsáveis por cada time. } \\
\text { Esses profissionais definem, com a equipe, a programação de atividades, } \\
\text { discussões técnicas e o estabelecimento de marcos e objetivos alcançados } \\
\text { semanais. }\end{array}$ \\
\hline $\begin{array}{l}\text { Resolução de } \\
\text { problemas, testes e } \\
\text { protótipos }\end{array}$ & $\begin{array}{l}\text { O protótipo constitui como o meio principal para a validação do projeto. A } \\
\text { resolução dos problemas que envolvem os testes e protótipos é avaliada por } \\
\text { membros de cada área específica. O projeto prevê o desenvolvimento de três } \\
\text { protótipos que serão testados em animais e humanos, e devem cumprir a todos } \\
\text { os requisitos normativos. Teste intermediários em subsistemas estão sendo } \\
\text { realizados à medida que as peças e componentes são disponibilizados para uso. }\end{array}$ \\
\hline $\begin{array}{l}\text { Controle e revisão } \\
\text { da gerência sênior }\end{array}$ & $\begin{array}{l}\text { O controle e revisão de projeto são feitos, até o presente momento, por encontros } \\
\text { com todos os colaboradores a partir de um plano de projeto preliminar (PDR), } \\
\text { que avalia tecnicamente o PDP com o foco em garantir a sua operacionalidade. } \\
\text { Nesse ponto de controle e revisão se estabelece a arquitetura do produto para } \\
\text { satisfazer os requisitos, dentro do orçamento, com o cronograma pré-definido. }\end{array}$ \\
\hline $\begin{array}{l}\text { Correções em } \\
\text { tempo real e no } \\
\text { decorrer do projeto }\end{array}$ & $\begin{array}{l}\text { Os ciclos de correção são realizados diretamente pelas áreas responsáveis: } \\
\text { Mecânica, Eletrônica, Software, e Gestão. As correções de projeto são avaliadas } \\
\text { semanalmente de maneira pontual, por cada equipe de projeto, e de maneira } \\
\text { coletiva, com todos os participantes e colaboradores, em reuniões gerais de } \\
\text { projeto. }\end{array}$ \\
\hline
\end{tabular}

O framework de diagnóstico (Quadro 1) permite uma agilidade de trabalho, com o foco nos resultados, e no cumprimento dos requisitos normativos para o produto desenvolvido. O time de gestão presta o suporte necessário para o acompanhamento 
sistemático do projeto. O time de hardware é responsável pelo projeto do sistema elétrico, dos sensores, microcontrolador, e sistema de alarmes. O time de mecânica é responsável pela construção dos componentes físicos do ventilador, e o time de software acompanha o desenvolvimento da plataforma de comunicação com o usuário.

Como o projeto está em andamento, avaliaram-se as fases compreendidas no prédesenvolvimento e parte do desenvolvimento do produto. No Quadro 2 apresenta-se uma visão simplificada das fases do PDP do projeto, considerando a natureza do projeto. Como não existe uma formalização de todo o PDP pelo curto prazo, o projeto não ter finalizado, e existirem mudanças significativas que podem ocorrer, uma análise com maior profundidade de detalhes deve ser realizada posteriormente.

Quadro 2- Elementos do PDP do projeto e sua respectiva estrutura em fases.

\begin{tabular}{|c|c|c|c|c|}
\hline $\begin{array}{l}\text { MACRO } \\
\text { FASES }\end{array}$ & FASES & $\begin{array}{l}\text { UNIDADES } \\
\text { ORGANIZACIONAIS }\end{array}$ & $\begin{array}{l}\text { MÉTODOS E } \\
\text { TÉCNICAS }\end{array}$ & $\begin{array}{l}\text { DOCUMENTOS } \\
\text { GERADOS }\end{array}$ \\
\hline $\begin{array}{l}\text { Pré- } \\
\text { desenvolvi } \\
\text { mento }\end{array}$ & $\begin{array}{l}\text { Identificação de } \\
\text { oportunidades e } \\
\text { definiçõos } \\
\text { estratégicas }\end{array}$ & $\begin{array}{l}\text { Coordenador do projeto } \\
\text { Professores } \\
\text { Fundação da UnB }\end{array}$ & $\begin{array}{lr}\text { Análise } & \text { de } \\
\text { mercado e } & \text { de } \\
\text { editais para } \\
\text { financiamento }\end{array}$ & $\begin{array}{l}\text { Plano de trabalho } \\
\text { do projeto }\end{array}$ \\
\hline \multirow[t]{4}{*}{$\begin{array}{l}\text { Desenvolvi } \\
\text { mento }\end{array}$} & $\begin{array}{l}\text { Projeto } \\
\text { conceitual }\end{array}$ & $\begin{array}{l}\text { Time de gestão e testes } \\
\text { Time de mecânica } \\
\text { Time de hardware } \\
\text { Time de Software } \\
\text { Empresa parceira } \\
\text { Fundação da UnB }\end{array}$ & $\begin{array}{l}\text { Planilha de custos, } \\
\text { FMEA, Matriz } \\
\text { QFD, Scrum, } \\
\text { Modelagem CAD, } \\
\text { Design de telas, } \\
\text { Scrum }\end{array}$ & $\begin{array}{l}\text { Análise de } \\
\text { requisitos, } \\
\text { Arquivo de } \\
\text { gerenciamento de } \\
\text { risco, } \\
\text { Especificações } \\
\text { técnicas, Matriz } \\
\text { de requisitos } \\
\text { normativos }\end{array}$ \\
\hline & $\begin{array}{l}\text { Desenvolviment } \\
\text { o de protótipos }\end{array}$ & $\begin{array}{l}\text { Time de gestão e testes } \\
\text { Time de mecânica } \\
\text { Time de hardware } \\
\text { Time de Software } \\
\text { Empresa parceira } \\
\text { Fundação da UnB }\end{array}$ & $\begin{array}{l}\text { Scrum, } \\
\text { Modelagem CAD }\end{array}$ & $\begin{array}{lr}\text { Modelos } & \text { CAD, } \\
\text { modelagem } & \text { dos } \\
\text { circuitos } & \mathrm{e} \\
\text { software, itens de } \\
\text { bancada, } \\
\text { Relatórios } \\
\text { projeto }\end{array}$ \\
\hline & $\begin{array}{l}\text { Construção dos } \\
\text { protótipos }\end{array}$ & $\begin{array}{l}\text { Time de gestão e testes } \\
\text { Time de mecânica } \\
\text { Time de hardware } \\
\text { Time de Software } \\
\text { Fundação da UnB }\end{array}$ & $\begin{array}{l}\text { Scrum, } \\
\text { Modelagem CAD }\end{array}$ & \begin{tabular}{lr}
\multicolumn{2}{l}{ Telas de software, } \\
Fluxogramas de \\
operação \\
sistema do \\
controle, Modelos \\
CAD, \\
especificações de \\
compras
\end{tabular} \\
\hline & $\begin{array}{l}\text { Testes } \quad \mathrm{e} \\
\text { validação }\end{array}$ & $\begin{array}{l}\text { Time de gestão e testes } \\
\text { Time de mecânica } \\
\text { Time de hardware } \\
\text { Time de Software }\end{array}$ & $\begin{array}{l}\text { FMEA, } \\
\text { QFD }\end{array}$ & $\begin{array}{lr}\text { Plano de } & \text { testes, } \\
\text { Matriz } & \text { de } \\
\text { verificação } & \end{array}$ \\
\hline
\end{tabular}


Realizou-se a identificação das unidades organizacionais que participam das fases de desenvolvimento de acordo com a divisão do trabalho por área técnica. Basicamente o projeto é coordenado por um professor que angariou os recursos necessários para executá-lo e junto com os demais professores, coordenam, cada um, um time de alunos de graduação e pós, divididos por área técnica. É importante mencionar que a equipe de professores conta com dois professores de eletrônica, um de software, um de mecânica, um médico pneumologista e o coordenador do projeto da engenharia de produção e mecatrônica.

O Quadro 2 apresenta na primeira e segunda colunas respectivamente, as macro-fases e fases do projeto, considerando o estágio atual do plano de trabalho acordado com a agência de financiamento. Na macro-fase de pré-desenvolvimento, apenas uma fase de identificação de oportunidades e definições estratégicas ocorre, sendo centrada na figura do "coordenador do projeto". Basicamente, com base nos editais de financiamento contra o COVID, no mercado de ventilação mecânica e no avanço da pandemia no Brasil, são definidas estratégias e validadas com o grupo de professores. Como o financiamento é individual para o pesquisador e não para um grupo, a interface com a agência, sob o ponto de vista das responsabilidades que gera, é realizada pelo coordenador.

A fase de "projeto conceitual" ocorre até que as especificações técnicas sejam geradas para as áreas de pneumática, mecânica, eletrônica e software. Uma vez que o projeto é desenvolvido em time virtual, as principais técnicas são baseadas em modelos CAD e de design de telas que são apresentados para a equipe. Essas são as principais ferramentas para a geração de especificações técnicas do produto. Em paralelo, atividades específicas da equipe de gestão buscam desenvolver os requisitos técnicos do produto utilizando matriz QFD, além de análises normativas para a geração de um checklist de riscos a serem evitados.

$\mathrm{Na}$ fase de desenvolvimento dos protótipos as duas técnicas basicamente utilizadas são o modelo de gestão ágil Scrum, com reuniões diárias e sprints semanais e a modelagem CAD, tanto mecânica quanto eletrônica e de design das telas. Além das especificações técnicas do produto, detalhadas em modelos CAD eletrônicos e mecânicos, a modelagem dos circuitos pneumáticos e do sistema de controle são desenvolvidos. Nessa etapa foi realizada uma revisão do projeto preliminar (PDR) com a apresentação dos detalhamentos do modelo de negócio do produto, projeto pneumático, mecânico, eletrônico e de software para a empresa, e aspectos regulatórios e testes. Foram coletadas impressões e demandas para melhoria do projeto e aspectos construtivos em geral. Nessa etapa, para que fosse realizada a análise da etapa PDR, foram gerados relatórios detalhados do projeto. 
A fase de construção dos protótipos é intensiva no uso dos métodos ágeis baseados em Scrum e na utilização de modelos CAD. Nessa fase são desenvolvidos modelos construtivos do produto já com características operacionais. Em software, são desenvolvidas as telas de interface dos médicos e fisioterapeutas que utilizarão o produto. Essas telas são apresentadas à equipe em pequenos vídeos e discutidas por todos. Os fluxogramas de operação do sistema de controle são apresentados à equipe por meio de modelos de simulação e esquemáticos de controle. Os principais componentes são discutidos pela equipe, considerando especialmente aspectos de funcionalidades, confiabilidade e robustez. Modelos CAD da mecânica e eletrônica são elaborados e discutidos pela equipe para compartilhar as discussões e refinar os aspectos de interface, com especial impacto na mecânica. Nesta etapa, detalhe especial é dispensado à especificações de compras, pois os componentes e partes do produto são quase $100 \%$ comprados. A compra é realizada por meio da fundação de pesquisa da Universidade de Brasília.

A fase final do projeto é a de testes e validação. Essa etapa foi iniciada com a compra dos equipamentos necessários aos testes, a estruturação das bancadas de testes e o desenvolvimento de um plano inicial de testes. Ela está em andamento com o desenvolvimento de testes parciais, já avançados se considerarmos o desenvolvimento do software. Do ponto de vista de hardware, testes com sistemas específicos, como o acionamento das válvulas, sensores de oxigênio, pressão e fluxo, são realizados. A etapa atual é justamente a integração do sistema com os testes funcionais em pulmões mecânicos. Há uma programação para realizar testes em animais e humanos, para os quais foi iniciado o trâmite nos comitês de ética respectivos.

Um detalhe relevante do projeto é a participação a Fundação de pesquisa da UnB (FINATEC), a qual é interveniente do projeto junto à FAP-DF, Fundação de Apoio à Pesquisa do Distrito Federal, financiadora do projeto. Na fase inicial do projeto, de definição de estratégias e oportunidades, a FINATEC compôs um comitê consultivo que escolheu o projeto em questão para financiá-lo. Uma vez escolhido, a FINATEC passou a ser responsável pela gestão do projeto, do ponto de vista financeiro. Isso significa que em todas as fases do projeto, os pagamentos de pessoal, compras de materiais e componentes e ao final do projeto, sua prestação de contas, passa pela Fundação. Como a interface com a FAP-DF é realizada por intermédio da FINATEC, qualquer alteração contratual, como a introdução de equipamentos, ou o remanejamento de rubricas entre itens de custeio ou de investimento, são realizados de maneira tri-partite, ou seja, o pesquisador aciona a FINATEC que aciona a FAP- 
DF, e eventualmente, há reuniões entre as três partes. Essa sistemática implica um conjunto grande de atividades de interface e burocráticas com impacto significativo no tempo demandado para coordenar o projeto.

Enfim, na etapa de construção de protótipos fica ainda mais ressaltada a interface com a Fundação no sentido de que todos os itens eletrônicos, mecânicos, pneumáticos devem ser aquisicionados, recepcionados e patrimoniados pela Fundação antes de enviados para a equipe de projeto de fato utilizá-los para a montagem, integração e testes. Esse fluxo é bastante iterativo e a complexidade de um ventilador mecânico é alta, impactando significativamente em lacunas de conhecimento para a própria Fundação com relação ao trâmite de itens complexos e fluxo de desenvolvimento de produtos de alta tecnologia. Em uma análise comparativa, verifica-se que em seus mais de 30 anos de operação, o maior número de componentes eletrônicos que a Fundação já havia comprado para um projeto era menor que a metade da compra realizada apenas dos componentes eletrônicos necessários para os protótipos do ventilador, os quais, ainda, deveriam ser comprados no mercado internacional.

\section{CONCLUSÃO}

A associação de duas representações do PDP para o projeto do ventilador pulmonar de baixo custo permitiu avaliar a ocorrência de elementos previstos na literatura especializada, no que tange a gestão do desenvolvimento de produtos, e identificou características singulares de um projeto materializado a partir de uma parceria público-privada e por equipes virtuais.

Os resultados apresentados comprovaram uma estrutura similar aos projetos do tipo mecatrônico, com alta interação entre os atores do projeto na realização das atividades e uma estrutura organizacional pouco estratificada, que permite uma agilidade do trabalho, com foco nos resultados e prazos estabelecidos em edital, e no cumprimento dos requisitos normativos, que são bastante minuciosos aos produtos de saúde para suporte à vida. Diferentemente de uma estratificação bem definida do PDP de empresas já consolidadas, verificou-se que não há uma grande dependência da estrutura de decisão das operações no desenvolvimento do produto, dada a horizontalidade da estrutura do projeto do ventilador pulmonar.

Uma vez que o projeto é desenvolvido a partir dos times virtuais, as principais técnicas são baseadas em modelos $\mathrm{CAD}$ e design de telas que são apresentados semanalmente pelas equipes do projeto. A metodologia ágil baseada no Scrum adequou-se de maneira oportuna ao tempo reduzido do projeto e a partir dos seus pilares de transparência, inspeção e adaptação. 
O projeto do ventilador pulmonar desenvolvido por meio colaborativo entre universidades brasileiras, a FAP-DF, a FINATEC, e a empresa privada de produção mecatrônica, revela inúmeros desafios quanto à gestão do PDP, devido à quantidade de agentes e atores do projeto, a complexidade do equipamento desenvolvido, e pela natureza virtual das reuniões e comunicação dos times de projeto. Assim, o diagnóstico do PDP do projeto revela ser um instrumento imprescindível para uma gestão eficaz. Tal análise acompanhará a evolução do projeto em trabalhos futuros, sendo adicionadas as mudanças relativas ao progresso das fases e atividades.

\section{REFERÊNCIAS}

BARBALHO, S. C. M. Modelo de referência para o desenvolvimento de produtos mecatrônicos: proposta e aplicações. USP, São Carlos, p. 275, 2006.

BARBALHO, S. C. M.; ROZENFELD, H. Análise do processo de desenvolvimento de produtos de uma pequena empresa de alta tecnologia. ENCONTRO NACIONAL DE ENGENHARIA DE PRODUÇÃO, v. 24, 2004.

BRYSON, M.; CROSBY, B. C.; STONE, M. M. The design and implementation of Cross-Sector collaborations: Propositions from the literature. Public administration review, v. 66, p. 44-55, 2006.

CARVALHO, C. R. R. D.; TOUFEN JUNIOR, C.; FRANCA, S. A. Ventilação mecânica: princípios, análise gráfica e modalidades ventilatórias. Jornal Brasileiro de Pneumologia, v. 33, n. suppl 2, p. 54-70, 2007.

DE VASCONCELOS, M. C. R. L.; FEREIRA, M. A. T. A contribuição da cooperação universidade/empresa para o conhecimento tecnológico da indústria. Perspectivas em ciência da informação, v. 5, n. 2, 2000.

KAPUCU, N. Disaster and emergency management systems in urban areas. Cities, v. 29, p. 41-49, 2012.

LASTRES, H. M. M.; CASSIOLATO, J. E. Sistemas de inovação: políticas e perspectivas, 2000.

LEMOS, D. C. et al. A interação universidade-empresa para o desenvolvimento inovativo sob a perspectiva institucionalista-evolucionária: uma análise a partir do sistema de ensino superior em Santa Catarina. UFSC, Florianópolis, 2013.

NOVELI, M.; SEGATTO, A. P. Processo de cooperação universidade-empresa para a inovação tecnológica em um parque tecnológico: evidências empíricas e proposição de um modelo conceitual. RAI Revista de Administração e Inovação, v. 9, n. 1, p. 81-105, 2012.

PUGH, S. Total design: integrated methods for successful product engineering. Addison-Wesley, 1991.

QUAYLE, A.; GROSVOLD, J.; CHAPPLE, L. New modes of managing grand challenges: Cross-sector collaboration and the refugee crisis of the Asia Pacific. Australian Journal of Management, v. 44, n. 4, p. 665686, 2019.

ROZENFELD, H. et al. Gestão de projetos em desenvolvimento de produtos. São Paulo: Saraiva, 2006.

SANTORO, M. D.; CHAKRABARTI, A. K. Firm size and technology centrality in industry-university interactions. Research policy, v. 31, n. 7, p. 1163-1180, 2002.

VALENTE, L. Hélice tríplice: metáfora dos anos 90 descreve bem o mais sustentável modelo de sistema de inovação. Conhecimento \& Inovação, v. 6, n. 1, p. 6-9, 2010.

WANG, H.; QI, H.; RAN, B. Public-Private Collaboration Led by Private Organizations in Combating Crises: Evidence From China's Fighting Against COVID-19. Administration \& Society, p. 1-26, 2021. 
WHEELWRIGHT, S. C.; CLARK, K. B. Revolutionizing product development process: quantum leaps in speed, efficiency, and quality. New York: The Free Press, 1992.

YIN, R. Estudo de caso: planejamento e métodos. Trad. Daniel Grassi. Rev. Cláudio Damacena. 2 ed. Porto Alegre: Bookman, 2001. 